\title{
émulations
}

\section{Nicolas Marquis - Du bien-être au marché du malaise. La société du développement personnel}

\section{Quentin Froment}

Émulations - Revue de sciences sociales

2016, «Comptes rendus critiques, En ligne»

\section{Article disponible à l'adresse suivante}

https://ojs.uclouvain.be/index.php/emulations/article/view/7113

\section{Pour citer cet article}

Quentin Froment, « Nicolas Marquis — Du bien-être au marché du malaise. La société du développement personnel », Émulations, en ligne. Mise en ligne le 5 février 2016.

DOI : 10.14428/emulations.cr.015

Distribution électronique : Université catholique de Louvain (Belgique) : ojs.uclouvain.be

(C) Cet article est mis à disposition selon les termes de la Licence Creative Commons Attribution, Pas d'Utilisation Commerciale 4.0 International. http://creativecommons.org/licenses/by-nc/4.0/

Éditeur : Émulations - Revue de sciences sociales / Presses universitaires de Louvain https://ojs.uclouvain.be/index.php/emulations

ISSN électronique : 1784-5734

UCL PRESSES

UNIVERSITAIRES 


\title{
Nicolas Marquis - Du bien-être au mar- ché du malaise. La société du développe- ment personnel
}

\author{
Quentin Froment ${ }^{1}$
}

Recensé : Nicolas Marquis, Du bien-être au marché du malaise. La société du développement personnel, Paris, Presses universitaires de France, 2014.

Cet ouvrage de 212 pages est issu d'une thèse de doctorat en sociologie soutenue en 2012 et récompensée par le Prix Le Monde de la recherche universitaire. Nicolas Marquis y analyse une pratique sociale en plein essor : la lecture des ouvrages de développement personnel (DP). L'auteur s'étonne du succès du DP en librairie mais établit très vite une rupture épistémologique avec la vision du DP classiquement répandue dans les sciences sociales, qui en perçoivent le lecteur comme «naïvement satisfait mais incapable de cerner les logiques objectives à l'œuvre» (p. 4). Dans cet ouvrage, il prend le pari qu'il est parfaitement possible de rendre le DP significatif et de ne pas le reléguer au rôle de témoin ou d'acteur d'une société sur le déclin. Pour cela, il remet le lecteur de DP au centre d'un processus au sein duquel interagissent le dispositif (l'ouvrage de DP) et la disposition de ce lecteur à lire ce type d'ouvrage. Pour cela, il s'appuie sur des données empiriques constituées de 55 entretiens compréhensifs réalisés auprès de lecteurs de DP et de 295 courriers de lecteurs adressés à trois auteurs de DP : Boris Cyrulnik, Thomas d'Ansembourg et Thierry Janssen.

L'auteur décompose son ouvrage en sept chapitres distincts. L'intention du premier chapitre est de proposer une remise en perspective de l'habituelle critique intellectuelle qui caractérise le DP comme symptôme et même acteur d'un malaise dans la civilisation. Il propose d'étudier les deux modalités majeures que prend cette critique : le « modèle du déclin » et le « modèle du pouvoir ». Le modèle du déclin (notamment porté par Rieff, Lasch et Senett dans les États-Unis des années 1960-1970 et par Castel, Gauchet et Lipovetsky dans le monde francophone) suppose que le succès du DP évoque le signe d'un affaiblissement de la société, lié à une poussée de l'individualisme et à une perte des liens sociaux. Ce que Nicolas Marquis critique dans ce modèle, c'est qu'il conduit à se retrouver face à des essais de théories sociales (qu'il nomme méta-psychologie), au centre desquels se trouvent de vagues hypothèses sur l'avènement d'un nouveau type d'individu (Narcisse) ou d'une nouvelle forme de société (culture du narcissisme). Le

${ }^{1}$ Doctorant en sociologie clinique au Laboratoire du changement social et politique, Université Paris-Diderot. 
modèle du pouvoir (porté principalement par Foucault), quant à lui, suppose que le succès du DP renvoie à une soumission du sujet contemporain à la norme de l'autonomie. Ici, le désinvestissement de la société et le repli sur la sphère privée ne sont plus perçus comme un déclin mais comme un maintien, voire une extension, de l'exercice du pouvoir et de la puissance des normes. Au nom de la liberté et de l'autonomie, les « techniques de soi » pousseraient les individus à agir sur eux-mêmes pour se constituer en sujets gouvernables. Moins virulente que pour le modèle du déclin, la critique de l'auteur porte néanmoins sur le même principe. Ici aussi, le DP est à la fois l'acteur et le pur produit de ce régime dans lequel il serait impossible de s'échapper. Or, selon l'auteur, si on veut vraiment se convaincre que les opérations réalisées par le DP sont spécifiques et méritent notre attention, il faut aller voir comment, concrètement, et dans quelle mesure le DP produit des individus gouvernables. Ce qui manque selon lui, c'est le personnage du lecteur, qui n'est acteur ni dans le modèle du déclin, ni dans celui du pouvoir. Il propose ainsi une théorie de la pratique afin de permettre à ces modèles de revêtir une certaine pertinence heuristique. Nicolas Marquis se pose alors les questions suivantes : que fait le DP à ses lecteurs ? Que dit-il de la société?

Pour répondre à ces questions, l'auteur élabore, dans le deuxième chapitre, un modèle d'analyse du DP adapté à une théorie de la pratique, en faisant parler le DP au sujet du monde dans lequel nous vivons, sans en passer par la difficile démonstration d'hypothétiques effets. Il s'attelle à cette tâche en articulant plusieurs approches théoriques. D’abord, les études sur la réception, c'est-à-dire les effets potentiels des contenus médiatiques que reçoit le public, en rapport avec les caractéristiques sociales et individuelles des récepteurs, le contexte social et historique dans lequel ils évoluent, ainsi que les stratégies d'interprétation qu'ils mettent en place. Ensuite, la reader-response theory qui vise à comprendre le processus d'engagement dans la lecture ou, en d'autres termes, comment le lecteur active un texte qui est à la base un objet inanimé. Enfin, et surtout, la théorie transactionnelle, qui propose une vision plus élaborée de la relation texte-lecteur en étudiant la transaction plutôt que l'interaction. Il s'agit, avec cette méthode, d'étudier le DP non plus uniquement comme un ensemble de signes, imprimés sur des pages, ou de messages que l'on disséquerait après les avoir extraits de leur contexte, mais bien comme une activité, une pratique, ou encore une expérience qui résulte de la connexion entre, d'une part, ce que l'auteur appelle un dispositif aux caractéristiques spécifiques - le livre qui propose et demande une implication à son lecteur - et, d'autre part, ce que l'auteur appelle une disposition dans laquelle se met le lecteur pour participer à la réussite de cette expérience à ses yeux. Ainsi, l'analyse ne porte plus uniquement sur le texte des ouvrages, mais également sur la pratique du lecteur en relation avec le texte.

Nicolas Marquis travaille spécifiquement, dans le chapitre 3, sur le dispositif, c'est-àdire sur l'analyse des textes, ce qui doit d'abord permettre de présenter le « monde » des ouvrages de DP. Il s'appuie sur la théorie transactionnelle dont l'objectif est de permettre au lecteur de voir quelque chose se modifier dans sa réalité entre le début et la 
fin de la lecture. Le livre de DP propose plusieurs façons de s'adresser au lecteur : (1) par la connivence passive du lecteur, qui suppose que le lecteur soit convaincu de la réalité des propos de l'ouvrage de DP, (2) par la coopération active, qui implique que, plus que de la réalité en général, l'ouvrage a pour ambition de parler du monde du lecteur, de ses relations sociales, de ses sensations, de ses pensées, de son parcours, et (3) par l'application de consignes, c'est-à-dire des instructions données plus ou moins explicitement par le texte au lecteur pour qu'il puisse développer une pensée, un sentiment ou une action qui dépasse le cadre de l'expérience de lecture.

Dans le chapitre 4, Nicolas Marquis se penche sur le travail du lecteur à proprement parler, c'est-à-dire sur la teneur des opérations que les individus réalisent lorsqu'ils lisent un ouvrage de DP, et la façon dont ils sélectionnent leurs propres réponses face aux stimuli des ouvrages. L'auteur affirme que les raisons pour lesquelles des personnes se mettent à lire des ouvrages de DP sont extrêmement variées mais qu'il existe, à chaque fois, un évènement dans le parcours du lecteur que l'auteur qualifie de «brèche de la vie quotidienne » : tout allait bien, et puis, tout à coup ou progressivement, " ça ne va plus ». Le DP va mettre en œuvre un changement dont le lecteur espère tirer satisfaction et réconfort, en procédant à une double ouverture : se soumettre à une révision pour que quelque chose change (estime de soi, hygiène mentale, comportement face à autrui, sens de la vie, etc.), et s'ouvrir à des supports dont on espère une aide. C'est très souvent dans ce cadre que le recours au DP est justifié et que cette double ouverture aboutit à la formulation d'une attente par rapport à celui-ci. La lecture de ces ouvrages n'est pas gratuite, mais plutôt de l'ordre de l'utilitaire, et implique un investissement important : en quoi cela répond-il à mes questions ? En quoi cela parle-t-il de mon problème ? En quoi ce qui m'est raconté va m'être utile ?

Le chapitre 5 décortique l'activité du lecteur en se référant aux trois idées majeures du chapitre 3, à savoir : la connivence passive, la coopération active et le respect des consignes mais, cette fois-ci, sous l'angle de la disposition. Tout d'abord, l'auteur cherche à savoir à quelles conditions les lecteurs acceptent de faire confiance à un livre de DP. Il doit être engageant (c'est-à-dire que le lecteur doit reconnaître une légitimité à l'ouvrage), crédible (avoir une assise suffisante pour pouvoir prétendre parler du monde de la vie quotidienne que le lecteur veut voir changer), attesté par une tradition passée, être prouvé par la science et, surtout, recueillir le témoignage du vécu de l'auteur du DP. Le lecteur va donc enquêter pour savoir si tel livre de DP est utilisable ou non, et, le cas échéant, il en vient à considérer qu'il peut se rapporter au monde présenté par le DP comme allant de soi. Cependant, il ne suffit pas que le livre soit suffisamment crédible, il est également attendu par le lecteur que l'ouvrage traite spécifiquement du monde qu'il connait empiriquement et voudrait voir changer (coopération active). Même si le lecteur sait que le livre de DP est destiné à des milliers de personnes, il le lit en faisant comme s'il traitait de sa propre vie. Enfin, le lecteur cherche à se transformer et à agir concrètement sur son nouveau monde grâce à de nouvelles com- 
pétences apprises. Les lecteurs parlent d'un «nouveau moi » dont les principales caractéristiques évoquent un rapport plus intense à la vérité de soi (ou à l'authenticité) et une puissance d'agir accrue.

Le chapitre 6 s'intéresse à ce que peuvent nous apprendre les observations récoltées dans les chapitres précédents sur le contexte socio-culturel de l'époque. Si le lecteur ne manifeste jamais d'étonnement, ni à l'égard de l'idée de travailler sur soi, ni sur les propos des ouvrages de DP, c'est parce que ces derniers parlent un langage qui possède de très sérieuses affinités électives avec le monde symbolique dans lequel ils vivent. Pour parler de l'environnement global dans lequel les lecteurs pensent vivre, ils se réfèrent à un ensemble de représentations socialement partagées dans les jeux de langage, notamment celui de l'intériorité, de la souffrance comme accès à la découverte de soi, d'une conduite de vie pour le travail sur soi et, enfin, d'une connotation positive du monde et d'une vision négative de la société. Ces jeux de langage permettent de donner un sens tant à l'acte même de lire un ouvrage pour effectuer un travail sur soi qu'à la vision particulière du monde et de l'individu que présentent les ouvrages de DP.

Le dernier chapitre est consacré à la signification sociale de la pratique de lecture du DP. Nicolas Marquis fait la liaison entre la pratique du DP et celle de la sorcellerie, toutes les deux marquées par une certaine attitude à la contingence, c'est-à-dire à la façon de traiter les infortunes et de chercher à attribuer des responsabilités. Mais là où celui victime de sorcellerie se demandera « qui m'en veut ?», le lecteur de DP préférera se demander en quoi il est responsable de sa situation. Dans une société où l'autonomie est transversalement vantée comme condition de l'existence, l'individu contemporain se doit d'allouer des responsabilités à son malheur et d'identifier des marges de manœuvre pour agir sur la situation ou sur ses conséquences. Le succès des ouvrages de DP est donc en ce sens révélateur du monde social dans lequel nous vivons actuellement. La signification sociale du DP trouve sa place dans la difficulté de l'individu contemporain à gérer l'injonction paradoxale d'être libre et autonome.

Le pari qu'il est parfaitement possible de rendre le DP caractéristique d'une époque, et non seulement le témoin ou l'acteur d'une société sur le déclin, est réussi dans cet ouvrage. Il s'empare avec justesse d'une pratique sociale des plus intéressantes, car en plein cœur des questionnements contemporains liés, entre autres, à l'idéologie du bonheur, du bien-être et de l'autonomie, mais aussi des plus complexes et des plus périlleuses, car située à l'interface entre différentes disciplines dont la sociologie, la psychologie ou la psychanalyse. S'éloignant d'un essai de théorie sociale comme on a (trop) souvent l'habitude d'en lire dans les travaux portant sur cette thématique, l'auteur réussit à faire une réelle démonstration sociologique. Il s'appuie pour ce faire sur des matériaux empiriques solides, en plaçant la disposition du lecteur de DP au centre de l'analyse sociale, fait assez rare pour être souligné. Le principal reproche que nous pouvons émettre à l'encontre de cet ouvrage concerne l'absence d'intérêt pour les caractéristiques sociales et genrées des sujets interviewés ( 42 femmes et 13 hommes), que l'auteur 
mentionne lui-même dans sa notice méthodologique : « Bien que je n’aie pas spécifiquement posé de questions portant sur la classe sociale ou la strate économique, les références que les individus font à leur mode de vie laissent à penser qu'ils appartiennent à une strate socio-économique moyenne voire supérieure [...] Cette absence d'individus issus de couches inférieures de la société peut bien sûr représenter un biais consécutif à la manière dont j'ai cherché à rencontrer les individus ». Ce n'est pas tant le fait que les individus interviewés soient issus en grande partie des classes moyennes voire supérieures ou que le nombre de femmes soit disproportionné qui nous pose problème, mais plutôt que l'auteur ne traite aucunement de la spécificité de ces données dans son ouvrage. En d'autres termes, il ne s'interroge pas sur le fait que les personnes qu'il a interviewées, ou dont il a recueilli des lettres, sont davantage des femmes et des personnes issues des classes moyennes et supérieures. Malgré cette critique, l'impression générale qui ressort de cette étude est qu'elle mène indéniablement vers une meilleure compréhension des faits sociaux contemporains, d'une part grâce à l'originalité d'un sujet souvent décrié dans les sciences sociales et, d'autre part, compte tenu de la méthode empirique employée qui place le lecteur de DP et le sens qu'il donne à sa lecture au cœur de l'analyse. 